\title{
Malignant minor salivary gland tumors: A retrospective study of 28 cases: Clinical experience of a single institution
}

\author{
Abeer Hussien Anter, Rash Hamdy Hamed \\ Department of Clinical Oncology and Nuclear Medicine, Faculty of Medicine, Mansoura University, Egypt
}

Received October 29, 2014; Revised December 21, 2014; Accepted December 29, 2014; Published Online January 01, 2015

\section{Original Article}

\begin{abstract}
Purpose: This retrospective study was done to report our experience with the management of patients with primary malignant tumors of minor salivary glands that were treated with various combinations of surgery, radiation, and chemotherapy and to review treatment outcome. Methods: The records of all patients with malignant minor salivary gland tumors presenting for treatment at our department between 2000 and 2010 were retrospectively reviewed. Variables were collected and outcome measures were defined in terms of overall survival, disease-free survival. Descriptive statistics were compiled and statistically evaluated. Survival was described using the Kaplan-Meier method. Results: Twenty eight patients (10 males and 18 females) ranging in age from 18 to 80 years (median, 64 years) met the criteria for inclusion in the study. The majority of tumors were located in the oral cavity $(n=20)$, followed by nasal cavity and paranasal sinuses $(n=8)$. Adenoid cystic carcinoma was the most common neoplasm $(\mathrm{n}=18)$. All patients were treated with surgery as the primary modality. Neck dissection was performed in $18 \%$ of patients, and all patients $(n=28)$ were treated with adjuvant external beam radiation therapy to a dose of 50 to $60 \mathrm{~Gy}$. The disease-free survival rate and overall survival at 5 years were $80 \%$ and $85 \%$, respectively. Conclusion: Postoperative radiation therapy is effective in preventing local recurrence in most patients with minor salivary gland tumors after gross total excision.
\end{abstract}

Keywords: Minor Salivary Gland tumours; Postoperative Radiation; Adenoid Cystic Carcinomas

\section{Introduction}

Minor salivary glands (MSG) are numerous (700 to 900) exocrine glands, which are distributed in the submucosa of the oral cavity, nasopharynx, tonsils, hypopharynx, and larynx. Heterotopic sites include lymph nodes, the thyroid gland, facial bones, and the hypophysis. ${ }^{1-3}$ Tumors of the intra-oral minor salivary glands constitute $2-3 \%$ of all malignant neoplasms of the upper aero digestive tract and less than $25 \%$ of all salivary gland tumors. ${ }^{2}$ Tumors arising at major salivary glands due to their well-defined anatomic borders and their higher incidence cannot be directly compared to minor salivary gland tumors. Most tumors arising at minor salivary glands are malignant. ${ }^{4,5}$

Surgery is the accepted primary modality of treatment for most of these tumors. ${ }^{6}$ The role of radiation in the post-surgical setting is less clear because there are few large series with long follow-up. Thus, the treatment approach to minor salivary gland tumors is based on principles of treatment of major salivary gland tumors, where a larger experience with postoperative radiation has established its value in patients with locally advanced disease; recurrent disease in an unirradiated site; high grade histological type; perineural spread; close, uncertain, or positive surgical margins; or neck nodal disease. ${ }^{7}$

These features are relatively more common in minor salivary gland tumors because they have a higher incidence of malignancy than do parotid tumors, adenoid cystic carcinomas are more common, and it is frequently harder to get clear margins, particularly in tumors arising from the paranasal sinuses. Thus, it is reasonable to expect that postoperative radiation would have a correspondingly greater role in their management. ${ }^{8}$ Malignant tumors of minor salivary glands are very rare. Therefore, design of management protocols is challenging. So our study that reports our experience in management of malignant MSG tumors beside other retrospective studies may help to design a protocol of management for those patients.

This study aims to retrospectively review our experience with the management of patients with primary malignant tumors of minor salivary glands that were treated with various combinations of surgery, radiation, and chemotherapy and to review treatment outcome, over ten years within a 
single radiotherapy (RT) unit in a large tertiary referral centre in our locality.

\section{Methods and Materials}

This study is a retrospective study of patients with primary malignant tumors of MSG that has been treated by post-operative radiotherapy at Clinical Oncology \& Nuclear Medicine Department, Mansoura University Hospital during the 10-year period (2000 to 2010).

A total 38 patients with primary malignant tumors of minor salivary gland were identified. The medical records of all patients with primary malignant tumors of MSG, were retrieved and reviewed. Demographic data, clinical characteristics, diagnostic investigations, histological typing, treatment modalities including type of surgery, radiotherapy and chemotherapy, follow-up, recurrence, and survival were noted.

All patients provided a history and underwent physical examination. Radiologic imaging included chest $\mathrm{x}$-ray, and computed tomography or magnetic resonance imaging scans. Computed tomography was preferred for cases with bone involvement, whereas MRI was reserved solely for soft tissue invasion.

\section{Radiotherapy}

All patients received their radiation therapy with or without cisplatin after a surgical procedure for removing a primary minor salivary gland cancer or after surgery for locally recurrent disease.

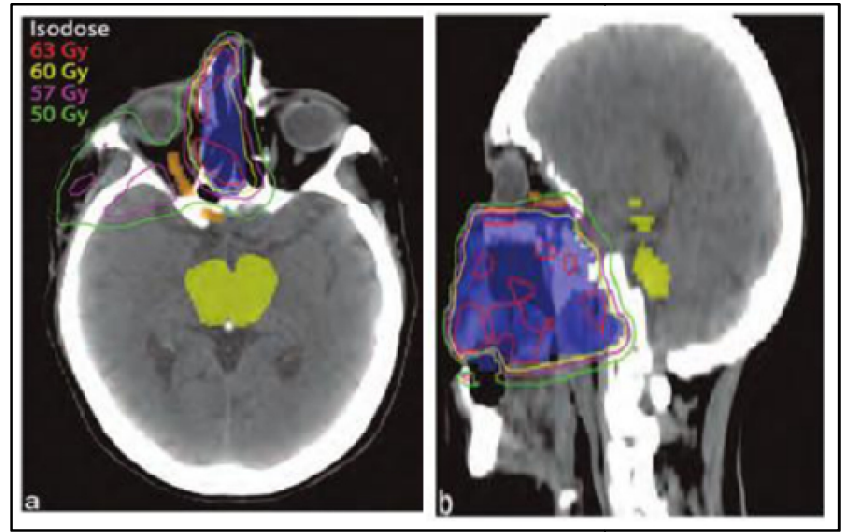

FIG. 1: A 58 year-old man with T4NOM0 Adenoid cystic carcinoma of Rt maxillary sinus, for post-operative radiotherapy to tumor bed only (nodal regions not electively treated). (a) Axial. (b) Sagittal images displaying isodose distributions. Shaded blue is PTV.

The interval between the date of last surgery and start of radiation therapy ranged from 2 to 8 weeks. Radiation techniques were dependent on the primary site. The techniques were grouped as follows: single appositional fields, parallel opposed fields, three field techniques, and wedged paired fields (Figure 1), with megavoltage equipment, using co- balt-60 gamma rays, $6 \mathrm{MeV}$ linear accelerator or 6-20 MeV electrons. Doses to the primary sites ranged from 5000 to 6000 cGy. The median number of fractions per course of treatment was 30. Concurrent chemotherapy consisted of cisplatin, which was typically administered weekly with a dose of $40 \mathrm{mg} / \mathrm{m}^{2}$.

\section{Statistical analysis}

The Kaplan-Meier method was used to estimate survival. Overall survival was defined as the interval between diagnosis and death or last follow-up. Disease-free survival (DFS) was defined as the interval between from the date of surgery to the earliest time of recurrence in local or distant sites, death from any cause or last follow-up.

A $p$-value $\leq 0.05$ was considered significant. All analyses were conducted with SPSS version 15.0 (SPSS for Windows, Rel. 13.0 2004. Chicago: SPSS Inc.).

\section{Results}

In the 10 -year period of 2000 to 2010,38 patients with minor salivary gland malignancy were treated in our institution, Patients with unresected or gross residual disease after attempted resection (6 patients) or with distant metastatic disease at the time of their radiation (4 patients) were excluded from the study. This left 28 patients between the ages of 18 and 75 years (median, 64 years) for inclusion in this analysis. Eighteen patients were female, and 10 were male.

The primary tumor site was in the oral cavity in 20 patients and in nasal cavity or paranasal sinuses in 8 patients (Table 1). The chief complaint on presentation was a lump (60\%), followed by an ulcerated lump (22\%). Overall $82 \%$ of the patients presented a lump with or without ulceration of the surface.

TABLE 1: Primary Site in 28 Patients With malignant minor sali-

\begin{tabular}{|c|c|c|}
\hline Site & No. of & Patients \\
\hline Maxillary sinus & & 2 \\
\hline Nasal cavity & & 4 \\
\hline Ethmoid sinus & & 2 \\
\hline Palate & & 4 \\
\hline Buccal mucosa & & 4 \\
\hline Lip & & 2 \\
\hline Tongue & & 2 \\
\hline Floor of mouth & & 4 \\
\hline Gingiva/alveolar ridge & & 2 \\
\hline Retromolar trigone & & 2 \\
\hline
\end{tabular}

Most patients (18) had a histologic diagnosis of adenoid cystic carcinoma. 6 mucoepedermoid carcinoma and 4 patients had adenocarcinoma (Table 2). Surgical procedures varied dependent on the size and site of the primary lesion. 2 patients had excisional biopsies, 14 patients had wide local 
excisions, and the remaining 12 patients had more extensive resections, including 3partial or total maxillectomies. After these procedures, 11 (39\%) patients had pathologic evidence of microscopic positive margins, and an additional 6 (21\%) had close (less than $5 \mathrm{~mm}$ ) or uncertain margins. A neck dissection was performed on 5 (18\%) patients, with pathologically positive lymph nodes being found in 3 patients. Thirteen patients (46.6\%) had pathological evidence of perineural invasion. Bone invasion by the primary was present in $6(21 \%)$ patients.

Table 2: Histological distribution.

\begin{tabular}{lcc}
\hline \hline Type of tumour & Number & $\%$ \\
\hline Adenoid cystic carcinoma & 18 & 64.3 \\
Mucoepidermoid carcinoma & 6 & 64.3 \\
Adenocarcinoma & 4 & 14.3 \\
\hline \hline
\end{tabular}

All patients received their radiation therapy with or without cisplatin after a surgical procedure for removing a primary minor salivary gland cancer. In 25 patients, radiation therapy followed the initial surgery; the remaining 3 (10.7\%) were irradiated after surgery for locally recurrent disease.

Local recurrence was recorded in two patients; one after 24 months, other after 5 years. Lung metastasis was only observed in three cases; one of them developed late lung metastasis after 5 years, as confirmed by biopsy from lung lesion. Also, three patients developed wide spread metastasis. Overall, 20 patients were free of local or disseminated disease at the last follow-up. Seven patients died during the course of our study. Two patients died due to unrelated causes (pneumonia, pulmonary embolism), three of them due to disseminated metastasis and the last two patients due to respiratory failure. The 5, 10 years overall survival rates for the whole series were $85 \%, 66 \%$ (Figure 2). On other hand, the 5,10 year disease-free survival was $80 \%, 64 \%$ respectively (Figure 3).

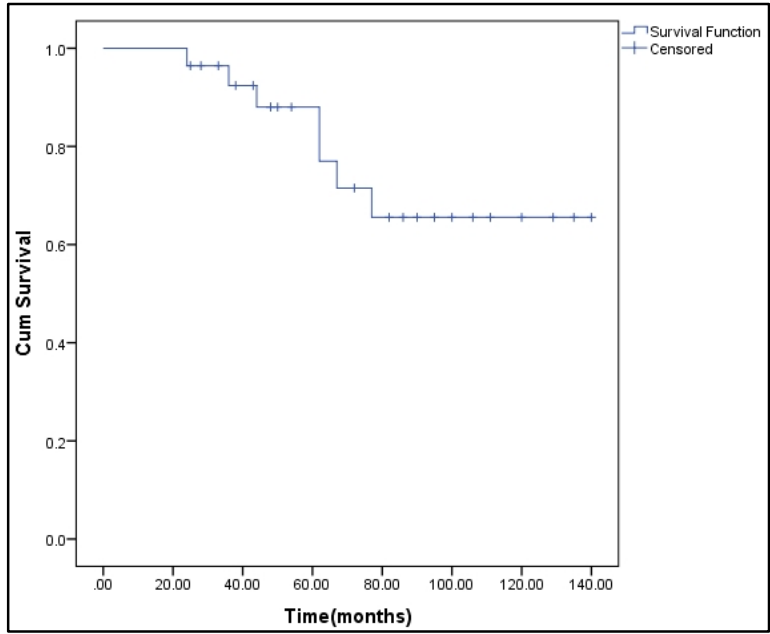

FIG. 2: Survival curve for 28 patients with malignant minor salivary gland tumors treated with postoperative radiation therapy.

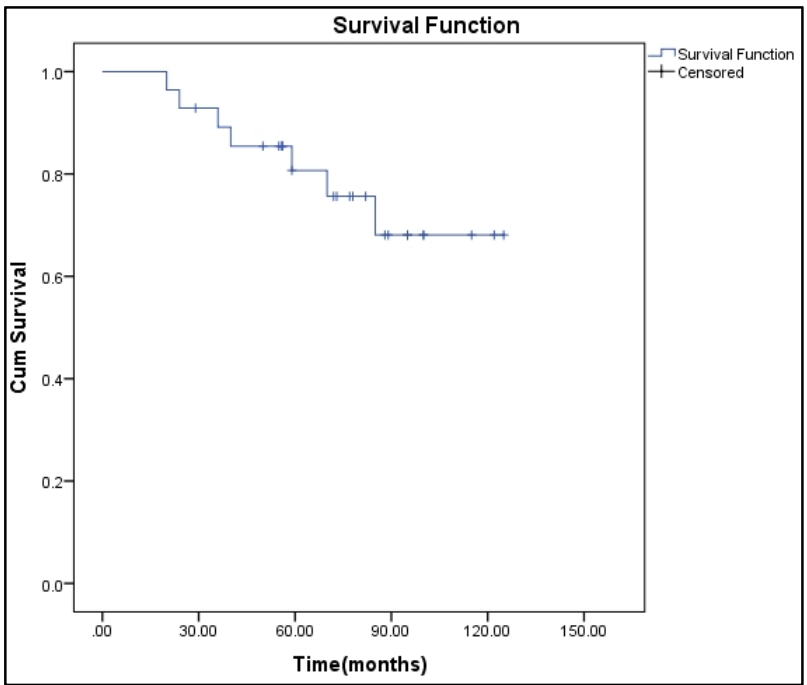

FIG. 3: Disease free survival curve for 28 patients with malignant minor salivary gland tumors treated with postoperative radiation therapy

\section{Discussion}

This study is a retrospective study of patients with primary malignant tumors of MSG that has been treated at Clinical Oncology \& Nuclear Medicine Department, Mansoura University Hospital. The present study revealed that primary malignant tumors of MSG occurred in the old population (median, 64 years). This was also reported by others. ${ }^{5,9,10}$

Patients were more often female than male in our data, with a ratio of 1.8:1. Review of the literature shows a female predilection among the patients with primary malignant tumors of MSG. ${ }^{5,11,12}$

According to other reports 5,13,14,15, the palate was the most commonly affected site, and this was also seen in our study. Moore et al. ${ }^{16}$ suggests that primary malignant tumors of MSG usually occurred at the junction of the hard and soft palates due to concentration of salivary glands in this region.

In this study, adenoid cystic carcinoma was the most common pathological type of primary malignant tumors of MSG (64.3\%) followed by mucoepidermoid carcinoma (21.4\%). Studies concerning west European population ${ }^{17-19}$ reported adenoid cystic carcinoma as the most common malignancy but according to other reports ${ }^{11,20,21}$; mucoepidermoid carcinoma was the most common malignant MSGTs.

In the present series, a neck dissection was performed on 5 (18\%) patients, with pathologically positive lymph nodes being found in $3(10.7 \%)$ patients which abides to the general proportion of 4 to $10 \%$ recorded in other studies $10,22,23$, Many factors usually dictate selective neck dissection, such as the site and size (T) of the tumor, the histological grade, and the age of the patient. Parsons et al. have classified MSG 
tumor sites according to the incidence of node positivity ${ }^{24}$, according to this classification; low incidence (less than 10\%) is observed in MSG tumors of the hard palate, nasal cavity, paranasal sinuses, lip, and buccal mucosa. The intermediate risk of positive neck at presentation should be expected in MSG tumors of the floor of the mouth and tongue. The higher risk for node metastases lies with MSG tumors of the pharynx and larynx. However, other authors advocate significantly lower lymph node infiltration than hematogenous metastases for MSG tumors. ${ }^{25}$

All patients received their radiation therapy after a surgical procedure for removing a primary minor salivary gland cancer. In 25 patients, radiation therapy followed the initial surgery; the remaining $3(10.7 \%)$ were irradiated after surgery for locally recurrent disease.

Adjuvant radiotherapy was offered to patients who presented with advanced stage, histopathologically proven positive neck metastases, close or positive margins and perineural invasion, Combination of radiotherapy and cisplatin was offered to patients with high grade histological subtype and advanced stage of the disease at presentation. Other authors reserve such a therapy for patients with distant metastases ${ }^{2}$, 26,27 or as palliative treatment. ${ }^{21,25}$

In this study, The 5 year survival rates for the whole series were $89 \%$ and this was also seen in another study., ${ }^{31}$ This trial has some limitations. One is the relatively small number of patients another factor is that it is a single institution study. Multicenter randomized Trials are necessary to clarify the role of radiotherapy in patients with MSG tumors.

\section{Conclusion}

Patients with minor salivary gland malignancies considered at high risk of local failure benefit from postoperative radiation. Local control rates are comparable to those reported for major salivary gland tumors, also late recurrences and metastases that were also noted in our study confirm the necessity of long-term follow-up of these patients, more than 5 years.

\section{Conflict of interest}

The authors declare that they have no conflicts of interest. The authors alone are responsible for the content and writing of the paper.

\section{References}

1. Parsons JT, Mendenhall WM, Stringer SP, et al. Management of minor salivary gland carcinomas. Int J Radiat Oncol Biol Phys 1996; 35:443-54.
2. Strick MJ, Kelly C, Soames JV, McLean NR. Malignant tumours of the minor salivary glands--a 20 year review. Br J Plast Surg 2004; 57:624-31.

3. Ord RA, Pazoki AE. Salivary gland disease and tumors. In: Miloro M, Ghali GC, Larsen PE, Waite $\mathrm{PD}$, associate, editor. Peterson's Principles of Oral and Maxillofacial Surgery, $2^{\text {nd }}$ ed. Hamilton, Ontario, Canada: B.C. Decker Inc. 2004; 671-7.

4. Wang D, Li Y, He H, et al. Intraoral minor salivary gland tumors in a Chinese population: a retrospective study on 737 cases. Oral Surg Oral Med Oral Pathol Oral Radiol Endod 2007; 104:94-100.

5. Toida M, Shimokawa K, Makita H, et al. Intraoral minor salivary gland tumors: a clinicopathological study of 82 cases. Int J Oral Maxillofac Surg 2005; 34:528-32.

6. Spiro RH, Thaler HT, Hicks WF, et al. The importance of clinical staging of minor salivary gland carcinoma. Am J Surg 1991; 162:330-6.

7. Garden AS, Weber RS, Ang KK, et al. Postoperative radiation therapy for malignant tumors of minor salivary glands. Outcome and patterns of failure. Cancer 1994; 73:2563-9.

8. Bell RB, Dierks EJ, Homer L, Potter BE. Management and outcome of patients with malignant salivary gland tumors. JOral Maxillofac Surg 2005; 63:917-28.

9. Lopes MA, Kowalski LP, da Cunha Santos G, Paes de Almeida O. A clinicopathologic study of 196 intraoral minor salivary gland tumours. JOral Pathol Med 1999; 28:264-7.

10. Papadogeorgakis N, Parara E, Petsinis V, et al. A retrospective review of malignant minor salivary gland tumors and a proposed protocol for future care. Craniomaxillofac Trauma Reconstr 2011; 4:1-10.

11. Yih WY, Kratochvil FJ, Stewart JC. Intraoral minor salivary gland neoplasms:review of 213 cases. J Oral Maxillofac Surg 2005; 63:805-10.

12. Wyszyńska-Pawelec G, Gontarz M, Zapała J, Szuta M. Minor salivary gland tumours of upper aerodigestive tract: a clinicopathological study. Gastroenterol Res Pract 2012; 2012:780453.

13. Pires FR, Pringle GA, de Almeida OP, Chen SY. Intra-oral minor salivary gland tumors: a clinicopathological study of 546 cases. Oral Oncol 2007; 43:463-70.

14. Dhanuthai K, Boonadulyarat $\mathrm{M}$, Jaengjongdee $\mathrm{T}$, Jiruedee K. A clinico-pathologic study of 311 intra-oral salivary gland tumors in Thais. JOral Pathol Med 2009; 38:495-500.

15. Barros CA, Silva Gurgel AC, Gomes CM, et al. Minor salivary gland tumors in a South American population. Arch Oncol 2010; 18:56-9. 
16. Moore BA, Burkey BB, Netterville JL, et al. Surgical management of minor salivary gland neoplasms of the palate. Ochsner J2008; 8:172-80.

17. Luukkaa H, Klemi P, Leivo I, et al. Salivary gland cancer in Finland 1991--96: an evaluation of 237 cases. Acta Otolaryngol 2005; 125:207-14.

18. Kokemueller H, Swennen G, Brueggemann N, et al. Epithelial malignancies of the salivary glands: clinical experience of a single institution-a review. Int $J$ Oral Maxillofac Surg 2004; 33:423-32.

19. Carino S, Cabrini RL. Meta-analysis of the literature on 1946 cases of minor salivary gland tumors of the palate. Acta Odontol Latinoam 2007; 20:23-31.

20. Buchner A, Merrell PW, Carpenter WM. Relative frequency of intra-oral minor salivary gland tumors: a study of 380 cases from northern California and comparison to reports from other parts of the world. J Oral Pathol Med 2007; 36:207-14.

21. Poomsawat S, Punyasingh J, Weerapradist W. A retrospective study of 60 cases of salivary gland tumors in a Thai population. Quintessence Int 2004; 35:577-81.
22. Jansisyanont P, Blanchaert RH Jr, Ord RA. Intraoral minor salivary gland neoplasm: a single institution experience of 80 cases. Int J Oral Maxillofac Surg 2002; 31:257-61.

23. Hyam DM, Veness MJ, Morgan GJ. Minor salivary gland carcinoma involving the oral cavity or pharynx. Aust Dent J2004; 49:16-9.

24. Parsons JT, Mendenhall WM, Stringer SP, et al. Management of minor salivary gland carcinomas. Int J Radiat Oncol Biol Phys 1996; 35:443-54.

25. Licitra L, Grandi C, Prott FJ, et al. Major and minor salivary glands tumours. Crit Rev Oncol Hematol 2003; 45:215-25.

26. Stannard CE, Hering E, Hough J, et al. Post-operative treatment of malignant salivary gland tumours of the palate with iodine-125 brachytherapy. Radiother Oncol 2004; 73:307-11.

27. Kokemueller H, Swennen G, Brueggemann N, et al. Epithelial malignancies of the salivary glands: clinical experience of a single institution-a review. Int J Oral Maxillofac Surg 2004; 33:423-32. 